\title{
EL ISOMORFISMO EPISTÉMICO COMO RESPUESTA AL ARGUMENTO POR EL ERROR
}

\author{
RODRIGO LAERA \\ Sosiedad Argentina de Análisis Filosófico (SADAF)
}

\begin{abstract}
RESUMEN: el objetivo de este trabajo consiste en presentar una respuesta al argumento por el error basada en el isomorfismo epistémico, donde la estructura explicativa que constituye nuestras creencias es similar tanto en el caso de que vivamos en el mundo real como en el caso que seamos, por ejemplo, cerebros en un cubeta. En una primera parte se plantean los problemas que suscita el argumento por el error (par., 2-4), mientras que, en una segunda parte (par., 5 y 6), se modifica el argumento de manera que se puedan eludir los problemas anteriores y se expone la respuesta isomórfica. Finalmente, se concluye que, dado el isomorfismo, optar por la posibilidad del mundo real o la posibilidad escéptica no tiene relevancia epistémica.
\end{abstract}

PALABRAS CLAVES: escepticismo; isomorfismo epistémico; argumento por el error; dogmatismo epistémico.

\section{Epistemic isomorphism in response to the argument from error}

ABSTRACT: The aim of this paper is to present a reply to the argument from the error based on the epistemic isomorphism, where the explanatory structure that constitutes our beliefs is similar in the case that we live in the real world as in the case we are, by example, brains in a vat. In the first part, the problems that arise from the argument from error are exposed (par., 2-4), while, in a second part (par., 5 and 6), this argument is modified so that it can be avoided the above problems and the isomorphic response is exposed. Finally, it is concluded that, given the isomorphism, to opt for the possibility of the real world or the skeptical possibility has no epistemic relevance.

KEY WORDS: escepticism; epistemic isomorphism; argument from error; epistemic dogmatism.

\section{PRIMERO: El ARgUMENTO POR EL ERROR}

La idea de que es imposible saber si los sucesos del mundo que nos rodean son reales radica en la dificultad de decidir entre explicaciones alternativas que justifiquen los datos de la experiencia. Dado que la evidencia disponible parece insuficiente para preferir una justificación entre otras, uno puede presentar alternativas radicales en forma de hipótesis. Algunas de estas alternativas suelen descansar en un cuerpo de creencias basado en la reflexión para concluir que la experiencia por sí misma no consigue justificarse $^{1}$. En cambio, otras alternativas suelen descansar en un cuerpo de creencias basado en algún aspecto de la propia experiencia, por ejemplo la percepción visual para concluir que la experiencia - al menos prima faciejustifica las creencias que provienen de ella ${ }^{2}$. Por supuesto, hay veces que las

1 Piénsese en las distintas variantes del internismo doxástico. Por ejemplo: LeHrer (1997); Wedgwood (2002); Conee \& Feldman (2004); o más recientemente Schafer (2014).

2 Piénsese en las distintas variantes del externismo doxástico. Por ejemplo: Goldman (1986); Bergmann (2006); o más recientemente WALKer (2016). 
explicaciones se complementan, echando mano de la noción de «contexto» o de «circunstancia» ${ }^{3}$. En consecuencia, habrá quienes afirmen que en contextos o en circunstancias especiales resultará imprescindible la reflexión si se quiere tener creencias justificadas acerca de algún aspecto del mundo, mientras que en otros contextos o circunstancias solo alcanzaría con la experiencia. Pero como estas alternativas no pueden ser confirmadas ni refutadas, lo más sensato sería no perder el tiempo con ellas y dedicarse a otros asuntos. Explorar los motivos de tal sensatez, considerando si estas alternativas son razonables, es el objetivo principal de este trabajo.

La anterior variedad de alternativas pueden entenderse como argumentos que median entre dos hipótesis radicalizadas: la del mundo real (MR) y la escéptica (ESC). Quien sostiene MR afirma que las creencias cotidianas acerca del mundo tienen una función explicativa que representan o se adecúan a hechos que sí existen, mientras que quien sostiene ESC niega tal afirmación. Lo interesante es que, siendo distintas, incluso contrarias, MR y ESC responden al mismo principio de subdeterminación (PS), que puede introducirse en tres pasos: (a) $S$ puede obtener una justificación para creer que MR, y siendo MR incompatible con ESC, la justificación supuesta de $S$ para MR favorece MR sobre ESC. Pero también (b) $S$ puede obtener una justificación para creer que ESC y siendo ESC incompatible con MR, la justificación supuesta de $S$ para ESC favorece ESC sobre MR. (c) No hay ningún dato independiente que beneficie a MR por sobre ESC ni viceversa.

El PS implica la falibilidad tanto de MR como de ESC y con ello la posibilidad de un argumento por el error. Así, suponiendo que tanto la creencia de ESC como de MR estén subdeterminadas, el argumento por el error afirma:

(i) si la justificación de $p$ mediante MR no es mejor que mediante ESC, y si tanto ESC como MR no son una instancia de conocimiento, entonces $S$ no sabe que $p$ ni mediante MR ni mediante ESC;

(ii) la justificación mediante MR no es mejor que la de ESC ni viceversa;

(iii) tanto ESC como MR no son una instancia de conocimiento;

(iv) por lo tanto, $S$ no está en disposición de saber que $p$ ni mediante MR ni mediante ESC.

Aceptando que PS conduce a las hipótesis radicalizadas de ESC y MR, cabe preguntarse cómo responder al argumento por el error y cuáles son las consecuencias epistemológicas de tales respuestas. Este trabajo está guiado por cinco problemas: el de la mejor explicación, que problematiza (i) y (ii); la respuesta dogmática a (iii) y una interpretación pirrónica de (iv); la idea de que (iii) podría no exigir fundamentos sin ser irracional al sostener tanto ESC como MR; la cuestión de que ESC y MR podrían referirse a mundos posibles, reformulando todo el argumento por el error mediante el uso del condicional subjuntivo; por último, la idea de que tanto ESC como MR pueden ser

3 Por ejemplo: Cohen (1999); Greco (2004); DeRose (2009). 
indistintamente instancias de conocimiento, en el sentido de que el dominio que abarca ESC no es epistémicamente diferente del que abarca MR.

\section{SEgUNDO: PROBLEMAS PARA UNA MEJOR EXPLICACIÓN}

Una de las claves del argumento por el error es que la primera parte de la conjunción de la premisa (i) y la premisa (ii) presuponen una variante del argumento a la mejor explicación, pues para que MR esté en igualdad de condiciones que ESC se debe considerar cómo sería que una de las dos fuera epistémicamente mejor. La idea principal aquí es que si una justificación es mejor que otra, entonces debe aceptarse. Sin embargo, no queda claro que quiere decir «epistémicamente mejor», ni por qué preferir racionalmente lo epistémicamente mejor ${ }^{4}$.

Por un lado, suponiendo que la idea de justificación envuelve la de explicación —donde «justificar» es «dar explicaciones»— el concepto de mejor puede ser interpretado como aquella explicación cuyos argumentos pretenden evitar las justificaciones ad hoc. Es decir, si ESC es ofrecida como una explicación de $p$, entonces ESC no debería estar aislada de otras explicaciones y datos que justifiquen $p$. En consecuencia, es posible interpretar ESC como una hipótesis en la que esto no se cumple, pues la justificación que uno tenga se encontrará no solo injustificada, sino aislada de explicaciones y datos. Por ejemplo, se puede decir que un mundo en el que uno es un cerebro en una cubeta no está conectado con otras cosas en ese mundo y que nosotros, aunque seamos cerebros en una cubeta, somos capaces de representar. Aunque esta interpretación parece plausible, pues la justificación es una cuestión normativa, no es la única interpretación aceptable. También se puede pensar que, todavía siendo un cerebro en una cubeta, uno está conectado con las mismas representaciones que no siendo un cerebro en una cubeta. Dicho de otro modo, se puede considerar que ESC y MR tienen el mismo alcance explicativo ${ }^{5}$. Para ello se precisa negar el disyuntivismo, es decir, negar la posición que sostiene que hay una diferencia entre las representaciones de quien habita en un mundo real y las de quien es un cerebro en una cubeta. Si no hay diferencia de contenido en

4 Se podría partir desde un punto de vista práctico y considerar que las consecuencias causales de las actitudes epistémicas justificadas son preferibles a las injustificados, porque reportan mejores resultados al tratar con el mundo. Sin embargo, esta consideración debe ser matizada, pues las consecuencias causales de tener una actitud injustificada no siempre son menos beneficiosas que las consecuencias de tener su alternativa justificada. Por ejemplo, un científico creyendo en contra de su evidencia que él se recuperará de una enfermedad, puede ayudar a una recuperación y contribuir así al crecimiento de los conocimientos mediante su investigación.

5 La motivación del disyuntivismo epistémico resulta del apoyo racional a la facticidad de un evento $E$, plasmado carácter relacional de la percepción de $E$, en tanto que comporta por sí mima evidencias sobre la creencia de $E$ en el mundo externo. Véase: McDowell (2011) y Pritchard (2012). 
ambas representaciones, entonces no habrá diferencias al enmarcar cada una de las explicaciones. En suma, debido a que estas dos interpretaciones parecen ser igualmente pertinentes, regresamos al problema de la subdeterminación ${ }^{6}$.

Por otro lado, si «epistémicamente mejor» se interpreta como «más simple», entonces en igualdad de condiciones una explicación más simple será superior a otra más complicada. Pero, así como antes no sabíamos qué era «epistémicamente mejor» ahora no sabemos qué es "más simple». La interpretación más inocente es que «más simple» quiere decir «menor compromiso ontológico». Sin embargo, esta interpretación no se ajusta a las demandas del argumento por el error, ya que lo que se justamente se desconoce con ESC y MR es la ontología del mundo. En consecuencia, uno no puede pensar en un menor compromiso ontológico si lo que se está cuestionando es el estatus epistémico de la propia ontología. Así, el argumento por el error demanda una decisión ontológica respecto a la cantidad de entidades representadas.

Siguiendo la línea de la simplicidad, hay un tercer aspecto a tener en cuenta: uno puede pensar que «mejor» implica alcanzar los objetivos epistémicos planteados. Así, desde un punto de vista pragmático se sugiere que, en lo que concierne a la explicación, más siempre es mejor. En cualquier caso, el desiderátum epistémico consiste en dar explicaciones que conduzcan a consecuencias deseables, como alcanzar mayor cantidad de proposiciones verdaderas que de proposiciones falsas. Pero justamente, lo que se discute es si ESC o MR son verdaderas o falsas, con lo que estaríamos ante una petición de principio. Aun si se tiene en cuenta este desiderátum epistémico, seguiríamos estancados en el argumento por el error. De hecho, las estructuras causales que justifican el contenido de ESC y de RM son idénticas; solo difieren en que las entidades se presumen falsas o verdaderas respectivamente. Esto hace que el desiderátum epistémico no se vea cumplido en ninguno de los dos casos, incluso suponiendo que quienes suscriben ambas hipótesis tengan el ojo de un espectador informado por el contenido representado ${ }^{7}$.

No obstante, se pierde algo importante en las motivaciones detrás del argumento del escéptico: la capacidad de afirmar algo como verdadero que se encuentre más allá de su propia hipótesis. Y esta pérdida podría usarse para cuestionar (ii). Sucede que la diferencia entre ESC y MR es que el escéptico cuestiona la capacidad de los sujetos para leer el carácter «real»o intrínseco del comportamiento causal de las cosas, mientras que MR considera que tal capacidad, al menos en lo fundamental, no falla. La indecisión entre ambas hipótesis surge - en su forma más inocente- con el pensamiento inicial de que la experiencia de cualquier objeto familiar podría ser causada por algo

\footnotetext{
6 En este punto importa diferenciar el disyuntivismo epistémico del metafísico.

7 Por supuesto, uno puede afirmar que los fines epistémicos no consisten en alcanzar el mayor número de verdades disponibles que falsedades, sino que se puede partir de la hipótesis que el fin último epistémico puede ser la aceptación de una actitud proposicional o la corrección. Sin embargo, este debate excede el marco del presente trabajo, para una presentación en torno a los fines epistémicos, véase por ejemplo VAHID (2009) o Hess (2010).
} 
distinto de ese objeto. De modo que la experiencia no fija necesariamente el carácter de la causa y, de esta manera, la premisa (ii) parecería mantenerse.

Finalmente, parece no haber una solución al problema de la mejor explicación para determinar qué elegir, si MR o ESC. Si ninguna de las dos hipótesis se define como «epistémicamente mejor», entonces ambas, con sus respectivas explicaciones, convivirán en el espacio lógico de la subdeterminación.

\section{TERCERo: El DOGMATISMO EPISTEMOlÓGICO Y LA ESTRATEGIA PIRRÓNICA}

Existe una estrategia escéptica que consiste en tomar el punto (iii) del argumento por el error para desarrollar una conclusión favorable. Esto permitiría dejar de lado (i) y (ii) con la respectiva vaguedad del término «mejor». La idea es sencilla y radica en que si ni ESC ni MR son instancias de conocimiento, entonces uno no puede pronunciarse acerca del tema. Por lo tanto, deberá suspender el juicio - o al menos lo más racional sería suspenderlo. La consumación de esta estrategia es el escepticismo pirrónico, que es justamente el desenlace de (iv). Así, para este tipo de escepticismo la evidencia acerca del mundo externo no es mejor que la evidencia de escenarios en el que somos cerebros en una cubeta, ni viceversa. Si bien ESC no gana la batalla dialéctica, mediante la estrategia pirrónica se puede retomar la conclusión del argumento por el error (iv), en la que $S$ no está en disposición de saber ni ESC ni MR.

¿Cómo responder a la estrategia pirrónica? El pirrónico carga su estrategia sobre (iii), y la respuesta más natural es negar (iii), que es lo que hace MR. Uno de los consecuencias de negar (iii), para simplemente sostener que MR es un conjunto de instancias de conocimiento, es el riesgo de volverse dogmático. Es un riesgo porque ya no se refutaría al escéptico en sus propios términos, es decir, no se podría creer o saber justificadamente que $p$, utilizando sólo las premisas que el escéptico nos permite usar ${ }^{8}$. En cambio, MR puede resignarse, y abandonar en parte la batalla dialéctica, para simplemente afirmar que se puede justificadamente creer y saber que $p$ sin contradecir hechos obvios sobre la percepción. De este modo, negando (iii), también se niega la conclusión (iv) y con ello el argumento por el error queda desmantelado.

$\mathrm{Si}$, como MR sugiere, el hecho de tener percepciones es suficiente para saber que son reales, entonces las intuiciones preteóricas de las experiencias perceptivas, no incluyen el empleo de explicaciones que justifiquen la experiencia perceptual. MR parecería ser silenciosa. Supóngase que MR fuera preteórica y no necesitara justificarse, entonces ¿por qué la experiencia perceptiva sería diferente de una mera imagen visual o una imagen que cualquiera de nosotros podría tener con los ojos cerrados? El defensor de MR podría apoyarse en Hume y decir que difieren en su fuerza y vivacidad, o en su aparente independencia de la voluntad del sujeto.

8 Esto es lo que Pryor (2000) llama proyecto anti-escéptico ambicioso, en contraposición del proyecto modesto. 
Incluso asumiendo que las anteriores diferencias existan, el escéptico podría continuar preguntando por qué las diferencias de grado deberían implicar una diferencia epistémica. Dada esta situación el escéptico pirrónico llevaría las de ganar, pues según ESC hay situaciones fenomenológicamente indistinguibles que falsean muchas de las creencias que MR toma como justificadas. Así, el escéptico reiterará que la percepción o la experiencia por sí sola no son suficientes para demostrar que las creencias implicadas por MR estén justificadas.

Los partidarios de MR podrían ponérsela más difícil tanto a ESC como al escéptico pirrónico, alcanzaría con apelar a una estrategia neo-mooreana y sostener que los estados doxásticos, por default, se basan en la confianza acerca de las entidades que se nos presentan. Y que, por lo tanto, el escéptico debe demostrar que esa confianza es injustificada y no MR que dicha confianza está justificada. Como el escéptico no puede demostrarlo sin caer en el dogmatismo, entonces tampoco sería lícito cuestionar el dogmatismo de MR. Lo que se le exige a uno, tiene que serle exigido al otro. Lo que se le está exigiendo al escéptico pirrónico es que justificadamente falsee las percepciones que, según MR, poseen correlato extensional. Pero esto solo lo puede hacer de tener la percepción necesaria para afirmar MR y también tener otra percepción necesaria para rechazar MR. Como ESC no puede dar con ninguna percepción que rechace MR (en todo caso las percepciones en un cerebro en una cubeta serían similares a las de mundo real), entonces ESC carece de fundamentos. Y, por lo tanto, no puede falsear justificadamente MR.

Esta posición conduce a que el defensor de MR no quede forzado al silencio, y pueda negar (iii) como era su intención desde el principio, donde una percepción daría una justificación para creer que $p$ si su contenido parece que $p$ y uno no tuviera ninguna razón para sospechar que cualquier alternativa escéptica a $p$ sea verdadera. Dicho a la manera del defensor de MR: las percepciones implicadas por MR no necesitan explicación alguna para estar justificadas, solamente es necesaria una explicación cuando la percepción se vuelve dudosa, estando bajo sospecha. Pero la sospecha debe tener alguna razón, uno no puede sospechar porque sí y pretender una defensa de dicha sospecha. ESC constituye esa sospecha, no es una razón para sospechar. Es decir, ESC es la sospecha. Pero una sospecha sin razón. Y es en esa sospecha en la que se basa también el escepticismo pirrónico antes presentado. Por lo tanto, si la hipótesis de ESC no posee razones que la avalen, entonces el escepticismo tampoco las poseerá, ya que este depende de la viabilidad de (iii).

\section{Cuarto: Sin EXPLICACIÓN O JUSTIFICACIÓN}

Negar la racionalidad de ESC apelando a la idea de que se trata de una sospecha injustificada parece ser un buen recurso, pues pone al escéptico entre las cuerdas. Pero veamos hasta dónde nos lleva y si es del todo plausible. 
Sin entrar en debate acerca de lo que hace que «S sea racional» o cuáles son los fundamentos o las propiedades de la razón, cabe destacar que la racionalidad epistémica no implica necesariamente justificación. Por ejemplo, $S$ podría ser racional al decir que ve una mesa roja delante de él y no una mesa blanca iluminada con luces rojas, sin dar una explicación o una justificación de porqué considera que no está iluminada con luces rojas. Distinto es cuando se trata de una acusación. Por ejemplo, si alguien me acusa de ser un asesino, espero una justificación y una explicación, aunque la acusación parezca absurda ${ }^{9}$. Del mismo modo, si alguien me acusa de irracional, también espero una explicación o una justificación, aunque haya acusaciones más graves que otras. Esta última es, justamente, la demanda de quien defiende MR. Si el escéptico acusa a $S$ de sostener proposiciones sin fundamento, entonces debería exigir una explicación al respecto. Como dijimos, esa explicación recae en la sospecha de que, por ejemplo, podemos ser cerebros en una cubeta. Sin embargo, también como se ha venido afirmando, esta sospecha, siendo parte de la acusación, debe estar justificada de algún modo. Volviendo al caso del asesinato, alguien dice que soy el asesino porque sospecha de mí y sospecha de mí porque pude haber estado en la escena del crimen. Por lo tanto, me invita a probar que no estuve en la escena del crimen, porque de lo contrario seré el asesino. Obviamente, este argumento no es tan sólido como para presentarlo delante de un jurado. Podría no saber cómo demostrar que no estuve en la escena del crimen y no ser el asesino, sin que nadie pueda demostrar que sí estuve. De la misma manera que parece irracional a quienes sostienen que MR carece de fundamento sin demostrar por qué MR es sospechosa.

¿Cómo puede responder el escéptico a este tipo de planteos para volver a sostener (iii)? Quienes defiendan ESC o el escepticismo pirrónico pueden comenzar sugiriendo que no hacen ninguna acusación. Pueden afirmar que una acusación enviste un carácter valorativo y que la sospecha no tiene ese carácter. Si esto es aceptado, el escéptico avanzará afirmando que MR es una hipótesis epistemológica y el estatus de una disciplina como la epistemología exige que las proposiciones que la constituyen estén basadas en argumentos.

Llegados a este punto se empieza a nadar en las convulsionadas aguas del contextualismo. Y una consecuencia del contextualismo —en sus más diversas facetas-es que el escepticismo no es completamente rechazado por MR, sino que es reducido o aislado al ámbito de las atribuciones de conocimiento, donde los estándares de evaluación son lo suficientemente exigentes. No obstante, aislar a ESC o darle un ámbito de pertenencia no es el objetivo de quienes afirman MR. El objetivo consiste en mostrar que, mediante atribuciones normales, las entidades que constituyen el mundo sí hacen un verdadero trabajo explicativo. En cambio, el objetivo de las hipótesis escépticas consiste en mostrar que las entidades que constituyen lo que decimos que es el mundo están presumiblemente sustituidas por otras, haciendo un falso o mal trabajo explicativo.

$9 \quad$ Véase Kaplan (1991). 
Más allá de que el contextualismo pueda ser visto como una salida diplomática del pasaje de (ii) a (iii), todavía avanzaría un paso más hacia la idea de racionalidad en ESC si se diera una explicación que satisfaga los compromisos epistémicos de MR. Para esto se puede pensar que del mismo modo que la racionalidad de MR depende de su función, también lo hace ESC. Así, uno es capaz de entender que la función de ESC es la de hacer evidente la posibilidad de falsear constantemente las proposiciones acerca del mundo ${ }^{10}$. Si decimos que al ver una mesa roja, el escéptico falsea la proposición «veo una mesa roja» sosteniendo que puede ser blanca iluminada por luces rojas o, siendo más radical, que la mesa puede no existir porque podemos ser cerebros en una cubeta. ESC nos saca del ámbito de lo real y nos introduce en el ámbito de lo posible.

\section{QUINTO: MUNDOS POSIBLES Y EL ARGUMENTO POR EL ERROR MODIFICADO}

Si quien defiende ESC pretende adentrarse en el ámbito de lo posible, entonces el argumento por el error puede modificarse de la siguiente manera:

(i*) si la justificación de $S$ para MR no fuera epistémicamente mejor que la justificación de $S$ para ESC, y la creencia de $S$ que ESC o MR no fuera una instancia de conocimiento, entonces $S$ no sabría ni ESC ni MR;

(ii*) hay al menos un mundo posible en que la justificación de $S$ para MR no es epistémicamente mejor que la de $S$ para ESC;

(iii*) no podemos conocer cuál es ese mundo, por lo que ni la creencia que ESC es una instancia de conocimiento ni la de MR;

(iv*) si $S$ no está en disposición de saber ESC ni MR, $S$ no está en disposición de saber MR, pero no saber MR es justamente el fundamento de ESC;

$\left(v^{*}\right)$ finalmente, ESC se encuentra justificada por el desconocimiento de MR.

Hemos discutido los problemas del concepto de «epistémicamente mejor», pero vale una aclaración más, sobre todo en referencia a las premisas $\left(i^{*}\right)$ y (ii*). Ni siquiera si el mundo propuesto por MR es mejor que el propuesto por ESC, podemos saber o ser conscientes que es mejor. Es más, aun si en este caso «epistémicamente mejor» quiere decir que es más racional, los dos parecen estar constituidos bajo los mismos estándares de racionalidad.

Ahora bien, la idea principal del argumento por el error modificado es que no podemos saber si este es un mundo posible donde, cuando veo una mesa roja, realmente estoy viendo una mesa roja o me parece ver una mesa roja. En efecto, hay al menos un mundo posible en que la justificación de la proposición «veo una mesa roja» conduce a la verdad de dicha proposición. Pero no sabemos

10 Justamente, esta es la idea que tenía Descartes en relación a la función del genio maligno. 
si este es ese mundo. Tanto si viera una mesa roja como una que aparenta ser roja, no sé si soy un cerebro en una cubeta ni sé que vivo de acuerdo con MR tal como se sostiene con (iii*) y (iv*) ¿Qué decir ante semejante desconcierto?

Si siguiéramos una estrategia a la Nozick (1994), diríamos que si $S$ no estuviera justificado en ver una mesa roja, entonces no estaría justificado en creer que no soy un cerebro en una cubeta ${ }^{11}$. De modo que en los mundos en que no estoy viendo una mesa roja, debido a la circunstancia que soy un cerebro en una cubeta, creo equivocadamente que lo que estoy viendo es real. Así, no resulta complicado sostener $\left(\mathrm{i}^{*}\right)$ y $\left(\mathrm{ii}^{*}\right)$ sobre la base de que no sé si estoy viendo una mesa roja o no, porque para demostrarlo debo también demostrar que hay alguna condición necesaria del conocimiento que se cumple en el presente caso. Dado que no es posible demostrar que la condición de verdad del conocimiento no se satisface, el escéptico pirrónico puede apoyarse tanto en (ii) como (ii*) y en (iii*), dependiendo del matiz que quiera darle a su conclusión. Dicho de otra manera, la propuesta es que si $S$ sabe que $p$, entonces si $p$ fuera falso, $S$ no creería erróneamente que $p$. Mi creencia de que no somos cerebros en una cubeta no satisface la idea de que si ESC fuera falso, entonces yo equivocadamente creería que ESC es falso. Así no sé si ESC es falso. El mismo argumento se puede plantear con MR, llegando a la conclusión de que no sé si MR es falso. Como no sabemos en cuál mundo posible - ESC o MRes verdadero o falso, el escéptico pirrónico tiene una razón epistémica para suspender el juicio y ESC tendría una justificación.

No saber en qué mundo posible vivimos es similar a no saber en cómo es el mundo en el que vivimos. Pero esta similitud ¿tiene algún valor epistémico si hay un isomorfismo epistémico entre las propuestas de ESC y MR? Quizás habría que preguntarse primero cuál es la diferencia estructural y causal entre ESC y MR, para luego ahondar en su valor epistemológico. Así, el escepticismo pirrónico lleva las de ganar si hay alguna posibilidad de que ESC sea verdadera, no si lo es. Por lo tanto, la clave de MR es volver a atacar tanto (iii) como (iii*).

El de MR es un mundo en que estoy viendo una mesa roja y que no es blanca iluminada por luces rojas, ni soy un cerebro en una cubeta. Pero ESC sostiene que MR puede ser falso y yo vivir un mundo en el que soy un cerebro en una cubeta. Pero si MR fuera falso, yo no estaría pensando que vivo en un mundo en el que soy un cerebro en una cubeta. Pero ¿por qué no podría pensando? Porque el mundo que me lleva a pensarlo me conduce a proposiciones falsas, por lo cual la proposición «soy un cerebro en una cubeta» sería falsa. El problema surge porque el escéptico acepta un análisis causal del conocimiento. Si lo acepta, su tarea será demostrar que la creencia de que estoy viviendo en un mundo donde soy un cerebro en una cubeta no es de hecho causada por ese tipo de mundo. Lo mismo sucede si se asume una explicación fiabilista según en la que el conocimiento es una creencia que

11 No se discutirá acerca de la validez del condicional disyuntivo porque no es el objetivo de este trabajo. 
emana de un mecanismo fiable de formación de creencias. Asimismo, la tarea del escéptico consistirá en explicar que la creencia de que soy un cerebro en una cubeta no emana de dicho mecanismo. De lo contrario el argumento ni siquiera puede empezar.

6. SEXTO: ISOMORFISMO EPISTÉMICO, DESMANTELANDO EL ARGUMENTO POR EL ERROR

La ventaja de ESC consiste en que envuelve al escepticismo pirrónico, porque si MR no es mejor que ESC ni viceversa, entonces uno racionalmente debería suspender el juicio, lo que haría que ESC, al menos su parte pirrónica, estuviera mejor justificada que MR. Este es un argumento que puede parecer bastante sólido. No obstante, también puede pensarse que a razón de que ni ESC ni MR pueden considerarse justificadas, entonces tampoco podría justificarse el escepticismo pirrónico - siendo una parte de ESC. Con lo cual parece que estuviéramos ante una especie aporía epistémica.

Una salida podría ser, no considerar la disputa entre MR y ESC como un problema en torno a la justificación, sino como un problema en torno a la racionalidad. Es decir, abandonar el enfoque que por el cual MR o ESC deben estar justificadas e introducir la idea de que alcanza con el MR o ESC sean racionales; aceptar que al menos una concuerda más con los estándares de racionalidad — sean cuales fueren-que la otra. Si estamos en condiciones de evaluar que tanto MR como ESC tienen la misma estructura epistémica que las vuelve a ambas racionales, entonces sí haría que el escepticismo pirrónico fuera la decisión correcta, volviendo a (iv) falsa.

Si algo tienen en común ESC y MR es su estructura epistémica, llamemos a esto isomorfismo epistémico. Con más precisión: el isomorfismo epistémico consiste en que la estructura explicativa que constituye ESC es similar a la de MR, tratándose de un duplicado. Si veo una mesa roja, en MR la mesa que estoy viendo será real, mientras que en ESC será un duplicado de la mesa que parece real, pero no lo es. Esto sucede con cada una de las percepciones, de manera que no hay diferencia en cómo se justifican las proposiciones cuyo contenido son perceptuales en ESC y MR, unas justificaciones serán de primer orden mientras que otras —las de ESC— serán de segundo orden. La diferencia entre primer y segundo orden consiste en la localización ontológica de aquello que se nos hace presente. Dado que MR atribuye diferentes ubicaciones de las entidades que postula, ESC también atribuirá su correspondiente pseudoubicación a cada una de ellas. Por ejemplo, si mi vista es una fuente fiable de que hay una mesa roja delante de mí, mi supuesta vista será una fuente fiable de que hay una mesa roja delante de mí, en mi cerebro en una cubeta. De la misma manera que dos objetos físicos no pueden ocupar un mismo lugar al mismo tiempo en MR, en ESC tampoco dos objetos ocuparán la misma pseudoubicación al mismo tiempo, siendo un cerebro en una cubeta. En consecuencia, todo lo que es una fuente fiable para justificar la creencia de que $p$ es el caso en 
MR, es una fuente fiable para justificar la creencia de que p es el caso en ESC. Y por supuesto, si hay regularidad o causalidad en MR, también la habrá en ESC.

Si se parte del isomorfismo epistémico, entonces desde el punto de vista práctico no importa tener un conocimiento de primer o segundo orden, siempre que no seamos conscientes de ello. Pero ¿qué sucede desde el punto de vista teórico? Tomemos como ejemplo el problema del nuevo demonio: supongamos que hay un mundo en el cual $S$ es engañado por un genio maligno. El genio le causa a $S$ que tenga las mismas experiencias perceptuales que a nosotros, tal que son fenomenológicamente indistinguibles. Sobre la base de esas experiencias, $S$ forma gran parte de sus creencias. Uno diría que esas creencias son falsas sobre la base de que las percepciones son productos del engaño del genio maligno. Sin embargo, en el mundo de $S$ los procesos perceptuales no son fiables porque conducen masivamente a falsas creencias acerca del mundo, aunque mucha gente y el propio $S$ diría que dichas creencias están epistémicamente justificadas. Así, las creencias de $S$ están epistémicamente justificadas, contrariamente a lo que presupone fiabilismo clásico (véase: Cohen, 1984; Foley, 1985). En suma, las decisiones y las creencias racionales para un dominio dado serán también decisiones y creencias racionales en el dominio gemelo.

Ahora bien, el isomorfismo epistémico lleva a pensar que no importa con qué dominio estemos tratando, los resultados epistémicos — ya sea en lo que respecta al primer o segundo orden de nuestras decisiones y creenciasserán los mismos, pues ambos son de alguna manera autorreferenciales. Es decir, las consecuencias y los objetivos de los fines epistémicos en un dominio sirven solamente en ese dominio y no en el dominio gemelo. De este modo, uno no puede adscribir ESC dentro del dominio propio del MR (ni viceversa), sin caer en contradicción. El problema estaría en que no sabemos en qué dominio vivimos. Por lo tanto, parecería que estamos de acuerdo con (iv): $S$ no sabe que $p$ porque no sabe si está en el domino de MR o de ESC. Pero ¿en qué difieren MR y ESC como para considerarlos una elección epistémicamente excluyente y necesaria? ¿Esta incertidumbre tiene consecuencias epistémicas no triviales? Ambas preguntas remiten a la relación del hombre con las evidencias que posee para afirmar que $p$ existe. No obstante, dado que hay un isomorfismo entre las creencias o el conocimiento de que $p$ existe en MR o en ESC - el mundo real o el mundo de un cerebro en una cubeta-, entonces la evidencia en uno y otro caso será la misma. Supongamos que la percepción de $S$ es la evidencia que posee para creer que tiene dos manos en MR, también la percepción de $S$ será la evidencia que posee para creer que tiene dos manos en ESC. De esta manera, siendo la misma fuente, la evidencia es también la misma. Lo que cambia, no es la creencia de que posee dos manos, sino en dónde las posee (MR o ESC). Y $S$ no suspenderá el juicio en relación a si tiene dos manos, sino que podrá suspender el juicio en relación al dónde las posee. En el caso de las falsas percepciones, como pueden ser las ilusiones o los espejismos, la situación de $S$ no cambia, porque $S$ poseerá la percepción equivocada de que $p$ es real en MR como en ESC. Es decir, $S$ percibirá equivocadamente y tendrá la creencia de hay un oasis delante suyo en MR o 
ESC, lo que cambia siempre es dónde se encuentra la percepción sea cierta o no.

En consecuencia, de aceptar la relación isomorfa entre MR y ESC, los pasos (iv) y $\left(\mathrm{v}^{*}\right)$ del argumento por error resultan insostenibles, de igual manera que la estrategia pirrónica de suspender el juicio en relación con nuestras creencias, y así aceptar una variable de ESC. Cuando se puede suspender el juicio al decidir si está en MR o ESC sin afectar tanto sus evidencias como sus creencias sea cual fuere el dominio; sin haya ninguna consecuencia epistémica. Del mismo modo, uno podría adherir a ESC dentro de MR y a MR dentro de ESC sin que esto afecte epistémicamente a $S$.

\section{SÉPtimo: CONCLUSIÓN}

Se ha discutido un tipo de argumento por el error que funciona como inspiración de diversas posiciones escépticas —incluyendo la alternativa pirrónica. Atravesando los problemas de dicho argumento, a la par de sus posibles soluciones, se ha considerado una respuesta basada en el isomorfismo epistémico. Esta respuesta sostiene que la estructura explicativa que constituye nuestras creencias es similar tanto en el dominio de ESC como en el de MR, pues no ven afectadas la disposición epistémica de nuestras evidencias.

Así, dado el isomorfismo epistémico, puede responderse la pregunta con la que se iniciaba este escrito: $¿$ Es sensato preocuparse por vivir en un mundo real o no? En efecto, esta pregunta tiene unos matices metafísicos y éticos de los que en este trabajo no se ha dicho nada, pero sí tiene un claro matiz epistémico que, curiosamente, parece pertinente a toda discusión teórica apoyada en el problema o desafío escéptico. En consecuencia, la anterior pregunta puede replantearse del siguiente modo: ¿Es sensato preocuparse por el problema o desafío escéptico? A lo que sigue ¿qué consecuencia en nuestras prácticas epistémicas tiene el problema o desafío escéptico? El isomorfismo epistémico nos da una buena razón para considerar sensato la falta de preocupación, pues no se ven afectadas nuestras evidencias a la hora de atribuir conocimiento. $\mathrm{O}$ mejor dicho, porque nuestras evidencias o nuestras atribuciones de conocimiento no cambian por pertenecer al dominio de ESC o al de MR, la preocupación acerca de si pertenecemos a uno u otro para fijar nuestras prácticas epistémicas parece infundada. En este sentido, el desafío escéptico que se refleja en el argumento por el error no tiene relevancia epistémica cuando pretendemos tratar epistémicamente con el mundo.

Por último, se ha argumentado que en el desacuerdo entre quienes sostienen ESC y MR no hay espacio para el escepticismo pirrónico, pues implica una indecisión entre dos dominios que son relevantes. Dado que no hay ninguna decisión que tomar debido a la estructura isomórfica de ambos dominios, suspender el juicio acerca de a cuál de los dos pertenecemos es simplemente aquello que no pretende ser: un dogma. 


\section{BiBLIOGRAFÍA}

Bergmann, M. (2006). Justification Without Awareness: A Defense of Epistemic Externalism. Oxford: Oxford University Press.

Cohen, S. (1984). Justification and Truth. Philosophical Studies, 46, 279-295.

Cohen, S. (1999). Contextualism, skepticism, and the structure of reasons. Noûs, 33(13), 57-89.

Conee, E., \& Richard, F. (2004). Evidentialism: Essays in Epistemology: Essays in Epistemology. Oxford: Oxford University Press.

DeRose, K. (2009). The Case for Contextualism: Knowledge, Skepticism, and Context, Vol. 1: Knowledge, Skepticism, and Context. Oxford: Oxford University Press.

Foley, R. (1985). What's Wrong with Reliabilism? The Monist, 68(2), 188-202.

Goldman, A. (1986). Epistemology and Cognition. Cambridge: Harvard University Press.

Greco, J. (2004). A Different Sort of Contextualism. Erkenntnis, 61, 383-400.

Hess, M. (2010). Is Truth the Primary Epistemic Goal? Lancaster: Ontos.

Kaplan, M. (1991). Epistemology on holiday. The Journal of philosophy, 88(3), 132-154.

Lehrer, K. (1997). Self-trust: A study of reason, knowledge, and autonomy. Oxford: Clarendon Press.

McDowell, J. (2011). Perception as a Capacity for Knowledge. Milwaukee: Marquette University Press.

Nozick, R. (1994). The Nature of Rationality. Princeton: Princeton University Press.

Pritchard, D. (2012). Epistemological Disjunctivism. Oxford: Oxford University Press.

Pryor, J. (2000). The Skeptic and the Dogmatist. Nous, 34, 517-549.

Schafer, K. (2014). Doxastic planning and epistemic internalism. Synthese, 191(12), 2571-2591.

Vahid, H. (2009). Epistemology of Belief. London: Palgrave Macmillan.

Walker, M. (2016). Externalism, Skepticism, and Skeptical Dogmatism. The Journal of Philosophy, 113(1), 27-57.

Wedgwood, R. (2002). The Aim Of Belief. Noûs, 36, 267-297.

Sociedad Argentina de Análisis Filosófico (SADAF)

Consejo Nacional de Investigaciones Científicas y Técnicas (CONICET)

rodrigolaera@gmail.com

[Artículo aprobado para publicación en enero de 2019] 